\title{
Distance Perception in Immersive Virtual Environments, Revisited
}

\author{
Victoria Interrante ${ }^{1}$, Lee Anderson ${ }^{2}$ and Brian Ries ${ }^{1}$ \\ ${ }^{1}$ Department of Computer Science ${ }^{2}$ Department of Architecture \\ University of Minnesota
}

\begin{abstract}
Numerous previous studies have suggested that distances appear to be compressed in immersive virtual environments presented via head mounted display systems, relative to in the real world. However, the principal factors that are responsible for this phenomenon have remained largely unidentified. In this paper we shed some new light on this intriguing problem by reporting the results of two recent experiments in which we assess egocentric distance perception in a high fidelity, low latency, immersive virtual environment that represents an exact virtual replica of the participant's concurrently occupied real environment. Under these novel conditions, we make the startling discovery that distance perception appears not to be significantly compressed in the immersive virtual environment, relative to in the real world.
\end{abstract}

CR Categories and Subject Descriptors: I.3.7 [Computer Graphics]: Three-Dimensional Graphics and Realism.

Additional Keywords: egocentric distance perception, immersive virtual environments.

\section{Introduction ANd Previous Work}

Immersive virtual environments (IVE) technology has enormous promise as a tool for facilitating the process of architectural design. By enabling users to experience a 3D model of a virtual environment from a first-hand perspective, before it is actually built, one has the potential to enhance their experiential understanding of the metrics of the designed spaces. However, many recent studies have reported evidence of a marked compression of egocentric distance perception in immersive virtual environments presented via head-mounted display systems - in some cases up to $50 \%$ or more, relative to distance perception in the real world. Perceptual distortion of such magnitude could present serious problems for applications such as architectural design, in which the accurate perception of size and distance is assumed, and is likely to be critical. Motivated by the long-term goal of exploring the potential of immersive virtual environments for facilitating the process of conceptual design in architecture, we set out to conduct a series of experiments to further investigate the phenomenon of distance compression in IVEs, with the objective of gaining insight into possible methods for better facilitating veridical distance perception in these environments. In this paper we report our initial findings from these experiments.

Nearly all of the studies to date that have compared distance perception in immersive virtual environments with distance perception in the real world $[27,26,19,2,24,8,21,14$, et al. $]$ have found evidence that distances are perceived as compressed in the virtual environments. Although considerable efforts have been undertaken to identify the sources of these effects, indications of distance compression have persisted under a wide range of display and technology conditions. Previous studies have ruled out a significant role for a wide variety of physical factors, such as the limited field of view available in a head mounted display vs. in the real world [13,3], and although Willemsen et al. [25] have found evidence that issues related to the ergonomics of head mounted display systems may account for some of the apparent compression observed, an explanation for the larger portion of the effect remains wanting. In the most significant recent finding, Thompson et al. [21] have demonstrated that the "graphical quality" of the virtual environment also cannot account for the observed effect.

However, we note that in all of the previous studies on this question of which we are aware except one ([14]), the presented virtual environment has represented a space that does not correspond to the space within which the user is actually, physically present during the experiment. This raises the possibility that the problem of distance compression might have some of its roots in the cognitive interpretation of the visual stimulus. For instance, if participants are experiencing a cognitive dissociation between the virtual world that they are seeing and the real world that they are occupying, i.e. if they do not believe with unambiguous certainty that the two environments are one and the same, the resulting lack of presence might be having some effect on the participants' actionable interpretation of the distances that they are perceiving. Just as, when we watch a movie, we are happy to adopt the camera's point of view as our own, without considering how we might have assumed that position, it is possible to imagine that when we view an environment via twin displays that are placed in front of our eyes, we might not automatically default to the same metric interpretations about this artificially observed environment as we would about the real world - at least not until we can trust that the two are functionally equivalent. Hence, by assessing participants' perception of egocentric distances in a high fidelity immersive virtual environment that they can confidently accept as being a faithful representation of their actual, occupied space, we sought in the studies reported here to disentangle the potential effects of higher level cognitive influences on distance judgments from the potential effects of lower level perceptual factors related to the display itself, such as limited resolution, poor dynamic range, or lack of appropriate accommodation cues to distance.

There are many possible methods that can be used to assess a person's perception of distance. The conceptually simplest approach is to have people make verbal estimates of the distance between themselves and a target location; however numerous studies have shown that verbal reports are generally less accurate than action-based metrics $[15,12$, et al.]. The most commonlyused action-based metric for assessing egocentric distance perception is 'blind walking', and numerous studies have verified that people can accurately walk, at a brisk pace and without vision, to previously seen targets [e.g. 17]. Alternative actionbased metrics include 'triangulated walking' [7], which is slightly less reliable than blind walking but which can be used with relative accuracy to assess the perception of very long distances within restricted spaces, and 'blind throwing' [18], which has been successfully used to dispel concerns that the indications of distance compression that have been experimentally observed might be artifacts caused by participants subconsciously hesitating to confidently walk without sight for fear of bumping into an obstacle. Also, Plumert et al. [16] have recently introduced a new, subjective action-based metric for assessing the perception of very long distances without requiring any walking, in which participants use a stopwatch to indicate their estimated walking 
time to a target. Nonetheless, blind walking remains the most accurate, reliable, and commonly accepted metric for assessing perceived distances in spaces within which it is possible to directly traverse the indicated interval, and this is therefore the metric that we have chosen to use in our experiments.

\section{EXPERIMENT 1}

The two goals of our first experiment were: to investigate distance perception under circumstances where there was no possibility of cognitive dissonance associated with the presented virtual environment being different from the occupied real environment, and to see whether providing users with short-range haptic feedback about the presence, size, and spatial location of a real object in the virtual environment, in combination with allowing them a moderate amount of time to experience the presented virtual environment in the context of performing an engaging and reasonably enjoyable task, might together enhance users' ability to make accurate judgments of egocentric distance in the virtual environment.

\subsection{Method}

We used a within subjects design with two environment conditions: real world and virtual world, and two exposure conditions: baseline and post-VE experience. Distance perception was assessed via blind walking over three different fixed paths of lengths 10 feet, 20 feet and 30 feet. No feedback was given at any time during the testing phase of the experiment.

\subsubsection{Apparatus}

All testing took place in the Digital Design lab, located on the University of Minnesota campus. At the time of our first experiment, the approximate dimensions of this room were 30 'x32'. During the experiment, the paths to be traversed were indicated by fixed tape marks on the floor, which were oriented at right angles to the direction of the path and located at the paths' beginning and end. The tape marks were portrayed at the same locations in both the real and virtual environments. The three paths were non-overlapping and a minimum eight foot buffer distance was preserved between the end of any path and any obstacle in the environment such as a wall or bookcase. Participants were required to wear earplugs to prevent the acquisition of any ambient auditory cues, and, in the real world conditions, to wear a blindfold to avert any accidental acquisition of inappropriate visual input.

The virtual environment was modeled as a set of texturemapped polygons, with the texture maps obtained from a mosaic of digital photographs of the walls, ceiling and floor of the room. All floor and wall fixtures were faithfully represented in situ via the texture maps; however the relief associated with details such as door knobs, hinges, and curtains was not preserved. There was a minimal amount of furniture and computer equipment abutting two walls in the real room, and these were not included in the virtual model. Figure 1 shows three screen shots of the virtual room from various points of view similar to those that might have been seen by a participant wearing the head-mounted display. In the short-range haptic exposure condition, participants were required to use an immersive modeling system to replicate a simple virtual model located on a table that was present in both the real and virtual environments. Information about our modeling system is provided in [1] and will not be repeated here. Figure 2 shows a screen shot of the virtual room with the table and a virtual model.

The virtual environment was presented using the $\mathrm{nVisor} \mathrm{SX}$ head mounted display manufactured by nVis, which offers a separate $1280 \times 1024$ resolution image to each eye over a $60^{\circ}$ diagonal monocular field of view (about 2.2 arc minutes per pixel) with $100 \%$ stereo overlap. Foam blinders attached to the HMD blocked any peripheral vision of the external environment. The device weighed approximately $1 \mathrm{~kg}(2.2 \mathrm{lbs})$, and at the time of this experiment was attached by a set of $10^{\prime}$ cables to a video control unit which we had mounted on a short wheeled cart that could be pulled around by tugging on the cables. During the experiment, we provided relief to the head from both the weight of the cables and the tugging of the cart by having an assistant manage the cables for each participant, making their presence transparent. Figure 3 shows what this situation looks like in practice. The viewpoint used for the image generation was determined in real time according to input provided at a rate of about $500 \mathrm{~Hz}$ from a HiBall 3000 optical ceiling tracker manufactured by $3^{\text {rd }}$ Tech. Positional updates for the pointing device were provided at a similar rate. Figure 4 shows one of our participants using the pointer to construct a virtual model.
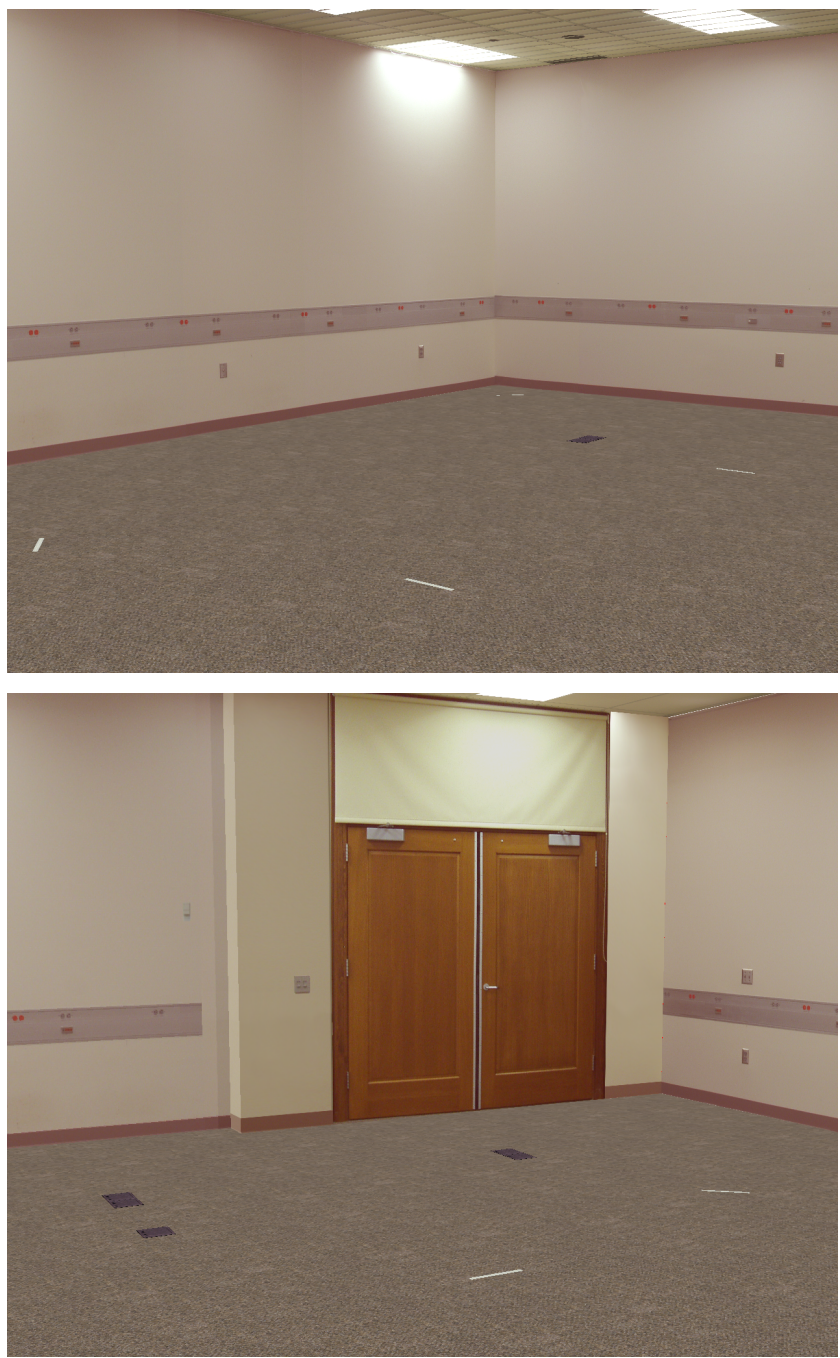

Figure 1: Screen shots of the virtual room model used in our first experiment. The black boxes on the floor are covered outlets for electrical and Ethernet connections. The white markings are texture mapped representations of the masking tape used to indicate the starting and ending locations of the target distance intervals.

\subsubsection{Participants}

In this first study, we collected data from seven naïve participants; three were female and four were male. Two were graduate 


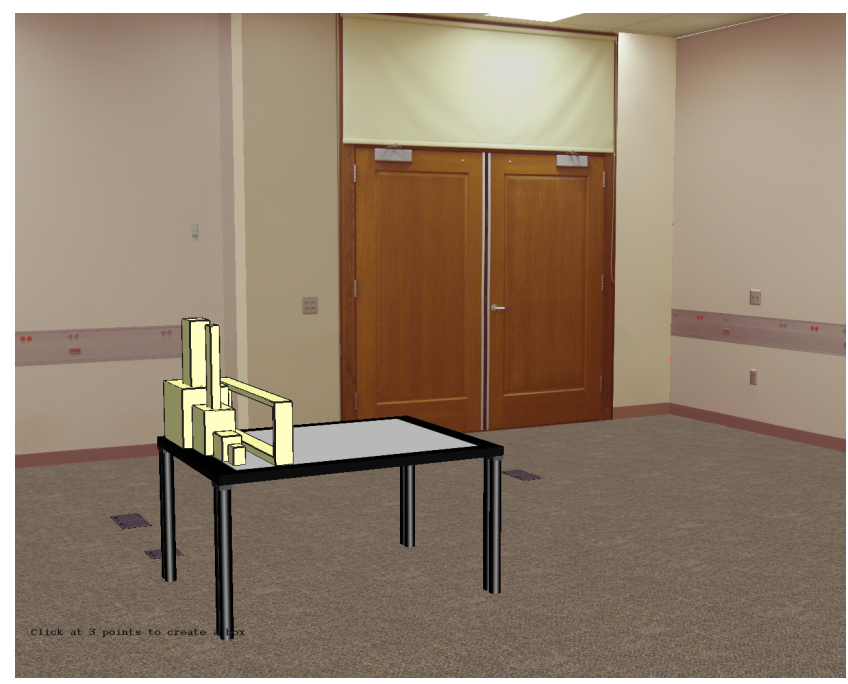

Figure 2: A screen shot of the virtual room in the short-range haptic feedback condition. During this portion of the experiment, a corresponding real table was present in the real room at the same depicted location.

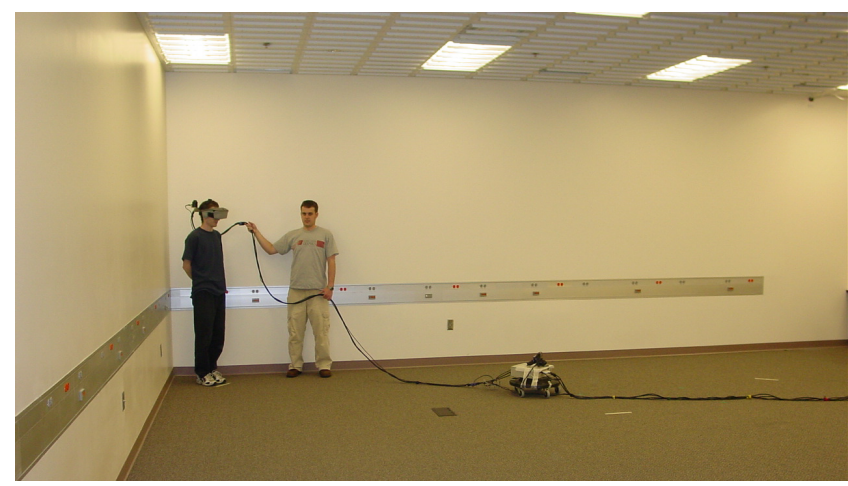

Figure 3: A photograph of a participant at the starting point of the $30^{\prime}$ distance interval wearing the head mounted display and tracking sensor, with an assistant managing the cables. The second sensor, which is not used during the walking trials, can be seen resting on top of the cart-mounted video control unit. Tape marks indicating the starting points of the 10' and 20' intervals can be seen on the floor to the right of the video control unit.

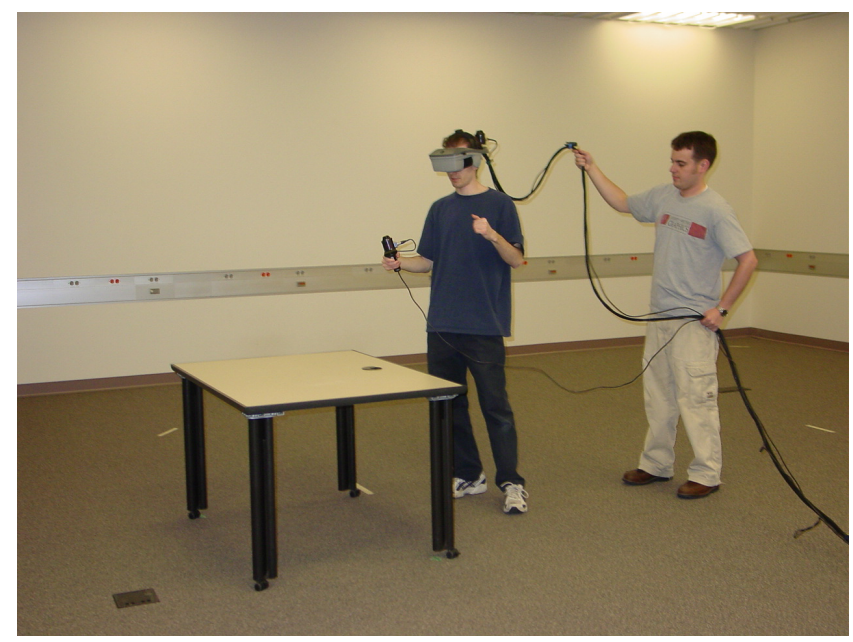

Figure 4: A photograph of a participant using the immersive modeling system in the short-range haptic feedback condition. students from the Department of Architecture, and five were graduate students from the Department of Computer Science. They were all recruited through informal contacts and compensated with \$20 Target gift certificates for their efforts. None of the participants had any significant IVE experience and all were naïve to the hypotheses underlying the study. The participants ranged in age from about 23-30, with an average age of about 25, and all had normal or corrected-to-normal vision. (Two of the participants normally wore glasses, and kept them on throughout all parts of the experiment.)

\subsubsection{Procedure}

Prior to testing, participants completed 15 practice walks, with feedback, in a basement hallway. Although previous studies had not found any significant impact on performance of prior practice, either with or without feedback [5], we decided to include a practice phase so that we could be certain that our participants were comfortable with performing the task, and that no one would be biased to 'walk short' out of caution. For consistency, the distances we used for the practice walks were the same as the distances we used in the testing. The procedure for the practice phase was similar to the procedure for the testing phase except that in the practice phase participants performed five consecutive walks over each distance $\left(5 \times 10^{\prime}\right.$ then $5 \times 20^{\prime}$ then $\left.5 \times 30^{\prime}\right)$, rather than performing them in interleaved order. Participants were instructed to position the end of their toes at the starting position, visualize the target distance, then close their eyes, cover them with the provided blindfold, and walk to where they thought the target location was. Although one early study [22] suggested a significant effect on performance of short term memory for a location, other researchers [e.g. 20] have not found this to be a significant factor. Therefore we did not attempt to enforce any particular amount of delay between when the visual stimulus was cut off and when the participant started walking. During the training phase, participants could remove the blindfold at the end of their walk to see how close they had come to the target. During the testing phase we guided participants in random circuitous paths around the room to the next starting location, in an attempt to disorient them and reduce the possibility of their getting any intuition about the accuracy of their performance. During the testing phase we collected four repeated measures from each participant for each distance interval under each condition. Within each condition, participants traversed the paths in interleaved order (first the $10^{\prime}$ path, then the $20^{\prime}$, then the $30^{\prime}$, then the $10^{\prime}$ again and so on).

The first condition tested was the baseline real-world condition. In this condition we measured elapsed time for each walk using a stopwatch, and we measured distance traveled from the marked starting position to a point aligned with the tips of the participants' toes but offset to one side. All of the participants conducted the training and baseline trials consecutively.

The second condition tested was the baseline VR condition. In this condition, participants were assisted in positioning their feet at the marked starting locations via verbal commands (since we did not have the means to track and therefore could not virtually represent peoples' actual foot locations). At the start of each trial, participants were instructed to look at the target, and then signal when they were ready. At the ready signal they were to close their eyes and walk to where they thought the target was. While one author managed the cables, another author sat at the controls of the computer driving the HMD and pressed keys to record the starting and ending positions and times for each walk (a single keypress recorded both position and time). Simultaneously with recording the starting position we would also black out the screen, to prevent any inadvertent acquisition of visual input by the participant while walking. A second keystroke recorded the 
stopping position but left the screen black, so that nothing would be given away even if the participant forgot to keep his or her eyes shut. A third keystroke was used to restore visibility after the assistant had guided the participant away from his stopping position, in a circuitous route, and brought him to the starting position of the next trial.

The third condition was the post-immersion VR condition. At the beginning of this phase of the experiment, a virtual table was displayed in the room, precisely registered with the location of a real table. A virtual model was displayed on the table, and after brief instruction in the use of our immersive modeling system, the participant was asked to spend 10 minutes working with the software to build a copy of the model on the table next to it, followed by 'free play' activities with the modeling system anywhere else in the room. Participants were encouraged reach out and touch the table, and to walk around to various different sides of the table while copying the model. If they began to walk in a direction that would cause the cables to become wrapped around the legs of the table they were requested to walk instead in the opposite direction. Our hypothesis was that haptic interaction with the real table might provide fairly robust cues to short-range egocentric and exocentric distances, so we included this condition in order to assess the extent to which participants might be able to use such information to correct any misperceptions they may have had about longer range distances in the IVE. Also, as previous research [9] had suggested that haptic interaction with real objects might enhance a participant's sense of presence in the IVE, we also sought, through this condition, to determine whether participants' distance judgments might become more accurate as they felt themselves to be more 'immersed' in the IVE. After 10 minutes of immersive design experience, the display was reset to remove indication of the table and all created models, and to restore indication of the distance markers on the floor. At the same time, the physical table was removed from the room and then the participant was asked to perform another 12 walking trials over the indicated distances.

The fourth condition was the post-immersion real world condition. Immediately after completing the trials for condition 3 , participants were assisted in removing the HMD, and, with their eyes still closed, were handed the blindfold and asked to repeat the walking trials one more time, this time in the real room. The purpose of this condition was to assess the extent of any adaptation that may have occurred as a result of the visual and haptic feedback that participants gained through their experiences in condition 3. Because we were concerned that any after-affects of adaptation to compressed distances in the IVE could disappear quickly, we were careful not to let participants engage in any sighted walking between the $3^{\text {rd }}$ and $4^{\text {th }}$ conditions.

Three participants completed the trials for all conditions consecutively on the same day. This typically required about 1.5 hours. Participants were encouraged to take rest breaks between each set of conditions except 3 and 4 . Two participants took a 1 or 2 day delay in between the baseline RW and baseline VR testing, and two took a 1 or 2 day delay between the baseline VR and post-immersion VR testing, for various scheduling reasons. There is no reason to believe that any of these variations in scheduling had any effect on the results.

\subsection{Results}

Figures 5-6 show the aggregate results of our first experiment, pooled over all participants. The error bars bound the $95 \%$ confidence interval. In Figure 5 we can see that there appears to be a slight amount of distance compression in each of the two VR conditions, increasing with target distance. The amount of compression appears to be slightly greater in the baseline VR

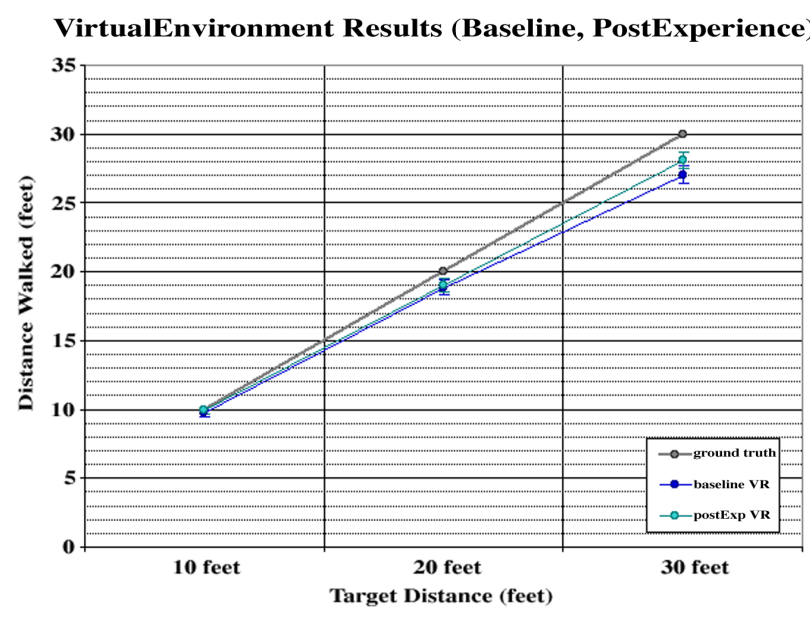

Figure 5: Distance walked vs. target distance across the two VR conditions, using pooled results from all participants.

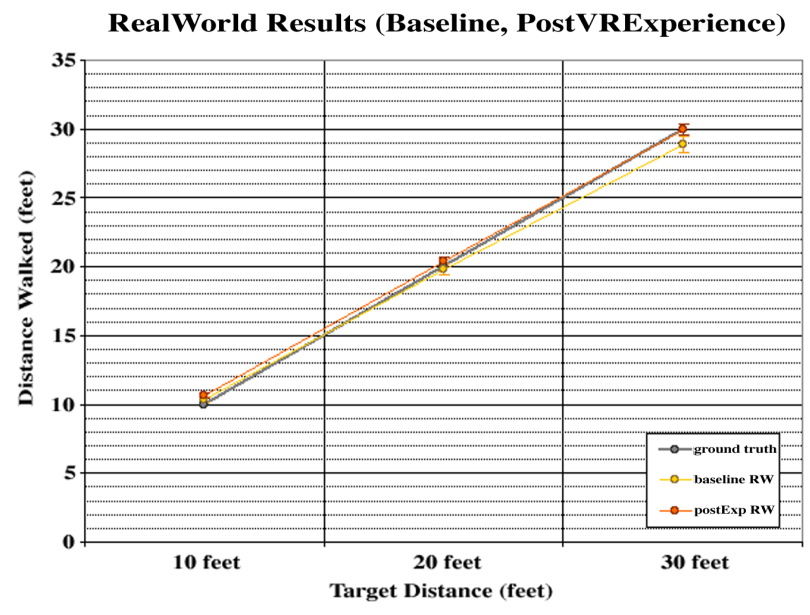

Figure 6: Distance walked vs. target distance across the two realworld conditions, using pooled results from all participants.

condition than in the post-immersion VR condition. What is striking, however is that the amounts of distance compression observed in both cases are remarkably small, particularly in comparison with the amounts of distance compression observed in previous studies. In figure 6 we can observe marginal indications of a slight overshoot in distance estimation, compared with the baseline, in the post-immersion real-world condition. Again, however, the magnitude of the effect is very small.

Figure 7 provides a close-in view of the differences in the traversed distances between the four experimental conditions. We see in the upper left chart that there is a small but statistically significant difference in the average distances traversed in the baseline VR condition, as compared with the baseline real-world condition, in conformation with previous findings that people tend to 'walk shorter' in immersive virtual environments presented via head mounted displays than they do in the real world. In the lower left chart we see that there is also a similarly small, but statistically significant, difference in the average distances traversed in the real world immediately after immersion for a period of time in the virtual environment, supplemented with haptic feedback, as compared to in the baseline real-world condition, supporting the hypothesis that participants are undergoing some adaptation to the compressed distance representation provided in the IVE and subsequently "walking 
longer' in the real world as an after-effect. On the lower right, we see that there is a small, but statistically significant, difference in performance in the blind walking task in the VR condition after the moderate period of immersion combined with some local haptic feedback, but for the longer range distance interval only. In the upper right chart we can see that this leads to achieving results in the IVE condition, for the longest-range interval only, that are effectively indistinguishable from the baseline results in the real-world condition.
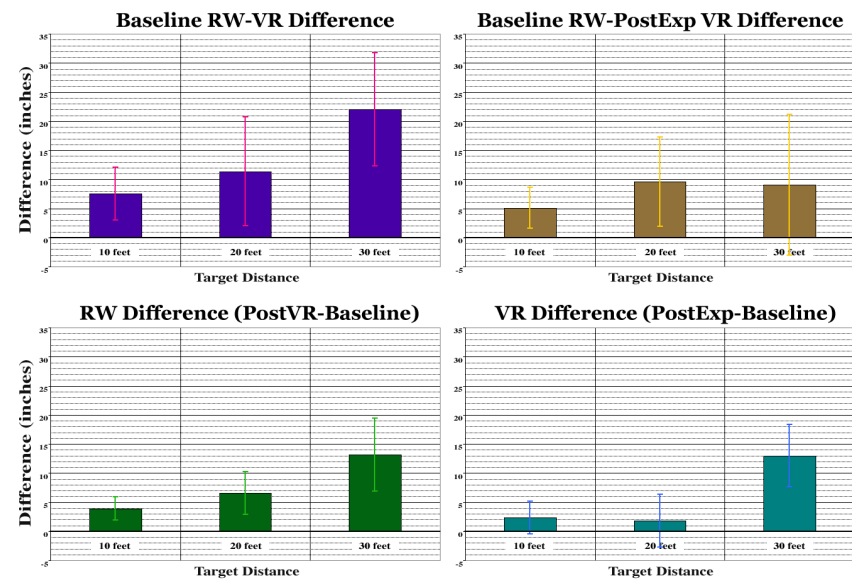

Figure 7: Differences in performance over various conditions.

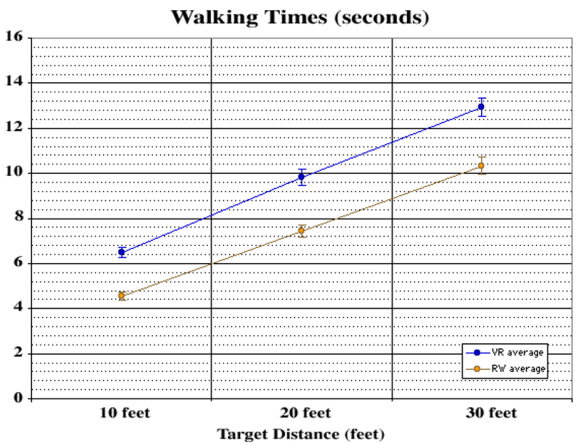

Figure 8: A comparison of the time taken to traverse the three distance intervals using blind walking in the real-world vs. in the virtual world.

In addition to considering the distances traversed, we also looked at the speed of walking in the different conditions. Figure 8 shows our findings. Participants traversed the three intervals at similar rates, but they walked significantly slower, overall, in the virtual environments conditions.

We also analyzed our results using ANOVA. We found significant main effects of participant $(\mathrm{p}<0.01)$, environment $(\mathrm{RW}$ vs. VR; $p<0.01)$, and distance interval $(p<0.01)$ on signed error rates (actual distance traversed minus target distance), but no significant main effect of experience (baseline vs. postimmersion) $(0.05<\mathrm{p}<0.1)$. We computed pairwise comparisons between participants using the Tukey's Honestly Significant Difference method, but we did not see anything illuminating in that analysis. Various subgroups of participants had statistically similar performance, and performance between all subgroups was highly overlapping.

Motivated by some concern about possible differences in spatial awareness between males and females, we then examined our results separately for the male and female participants. However, we did not find any significant differences in the results between these two groups. It should be noted that we did not intend to directly investigate the potential extents of any differences in spatial awareness between the sexes; this statistical test was conducted only to allay any potential concerns that our results might somehow be an artifact of an uneven balance between male and female participants. We conducted similar tests to rule out the potential of confounding effects due to background (architecture vs. computer science).

\subsection{Discussion}

The most interesting finding of our first study was that we observed a lower-than-expected amount of distance compression in the immersive virtual environments, in both the baseline and post-experience conditions. Our immediate interpretation was to view this discovery as a confirmation of the hypothesis that, by creating our virtual environment to be an exact replica of the users' concurrently occupied real environment, we had apparently removed one of the main factors that had contributed to the indications of distance compression that were found in the previous experiments by others, and we were very encouraged by the potential implications of this finding for the prospects of effectively using VR for architectural design applications.

However we were soon drawn to consider whether it might only have been some confounds in the details of our experimental procedure that were leading us to find so much less evidence of distance compression than was found in previous studies by other researchers. We identified two potential confounds. The first is that we conducted training, with feedback, over the same distance intervals that we used in the test conditions. Although previous studies that had specifically aimed to examine the effects of training with feedback, as opposed to training without feedback, on performance in blind walking trials found no significant effect from the provision of feedback [5], we noted that these studies had used intervals of different lengths in the test and practice phases, so there was no way to be certain that the effects of training would necessarily be equally benign in cases where intervals of the same length were used in both testing and training. Although we were compelled to consider the possibility that there could have been some motor learning effect from the training, we did not believe this to be likely, as previous studies examining the ability to reproduce distances walked, based on immediately recently received locomotor information alone, had found that participants were not able to accurately use this information, tending instead to overestimate short distances (2m) and underestimate longer distances (up to 10m) [11]. The second potential confound we identified in our first experiment was that we had our participants perform the same three walking tasks repeatedly over all of the different conditions. Therefore, we could not rule out the possibility that participants were undergoing some learning effects during the course of the experiment, even though they did not get any feedback about their performance. Hence, we would need to run some followup experiments to rule out these two potential confounds.

\section{EXPERIMENT 2}

The main objective of this followup experiment was to determine whether our main finding - that participants did not appear to experience profound distance compression in a high quality immersive virtual environment that they knew was an exact match to their actual occupied real environment - would continue to be maintained after we corrected for all of the potential methodological confounds.

\subsection{Method}

In this experiment, we used a mixed experimental design with two independent variables. As before, the within-subjects variable 
was environment: whether the distance judgments were made in the real world or in the virtual world. Handling this as a withinsubjects measure enabled us to establish a control level of performance for each individual. The between-subjects variable was exposure (whether distance judgments were made in the real world first or in the virtual world first). Participants did not go through any training prior to testing, and no feedback was given to any participant about his or her performance at any time.

\subsubsection{Apparatus}

As before, all testing took place in our lab, and used the same tracking and display equipment. However by the time of our follow-on experiment, the length of the cables connecting the head mounted display to the video controller had been increased to 15 ', and our room had been reconfigured to house a large, rearprojected, curved screen display, reducing the dimensions of the open space to 30 ' long x $25^{\prime}$ wide in the center, tapering down to $16.5^{\prime}$ wide at the edges. The length of the longest unobstructed diagonal was now about 34', limiting the maximum distance over which we felt that we could comfortably ask people to perform blind walking to $25^{\prime}$, in order to leave plenty of space between the end of the distance interval and any wall. Because of the changes to the real room, we were forced to create a new virtual room model, which, as before, represented a geometrically and photographically exact replica of the real environment, minus the furniture. Figures 9 and 10 show a photograph of the new real room and a screen shot of new virtual room taken from similar vantage points. In order to prevent the possible acquisition of auditory cues to location in the environment, participants wore a small portable radio that was tuned to play static.

In the course of our first study, we had noted that the head mounted display optics of the nVisorSX caused moderate pincushion distortion. For our follow-up study, we took steps to correct for this distortion using an approach similar to that described in [23]. What we did was to texture-map the contents of the frame buffer, in real time, onto a carefully calibrated convex surface, producing a barrel-distorted image. When this pre-distorted image was viewed through the head-mounted display, the equal but opposite distortions canceled out, enabling accurate perception of the true geometry of the scene.

\subsubsection{Participants}

We recruited ten new participants from the University of Minnesota and the Minneapolis community for this portion of the followup study. Their ages ranged from about 20 to about 45, and they included 4 females and 6 males. Four of these participants were undergraduate students from the Department of Architecture, two were graduate students from the Department of Computer Science, and the remainder were working professionals from the local community. Only one of the participants had previously been in to our lab, and he was assigned to the realworld-first exposure condition. All participants were compensated with \$10 Target gift certificates for their efforts.

\subsubsection{Procedure}

All participants, regardless of the exposure group to which they were assigned, began by entering our lab and filling out a consent form which explained the experimental procedure that would be followed. This process took about 2 minutes. Participants did not have an opportunity to walk around in the room prior to testing. Each participant was then asked to perform 30 trials of blind walking: 10 in the real world environment, and 20 in the corresponding high fidelity virtual model of that same environment. Half of the participants performed the real world trials first; the other half performed the virtual world trials first.

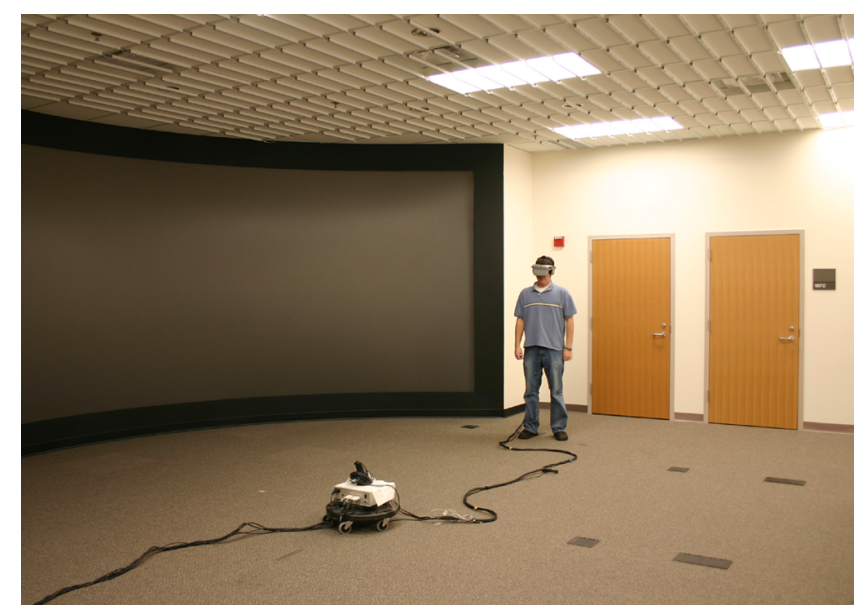

Figure 9: A photograph of the room environment used in our followup experiment.

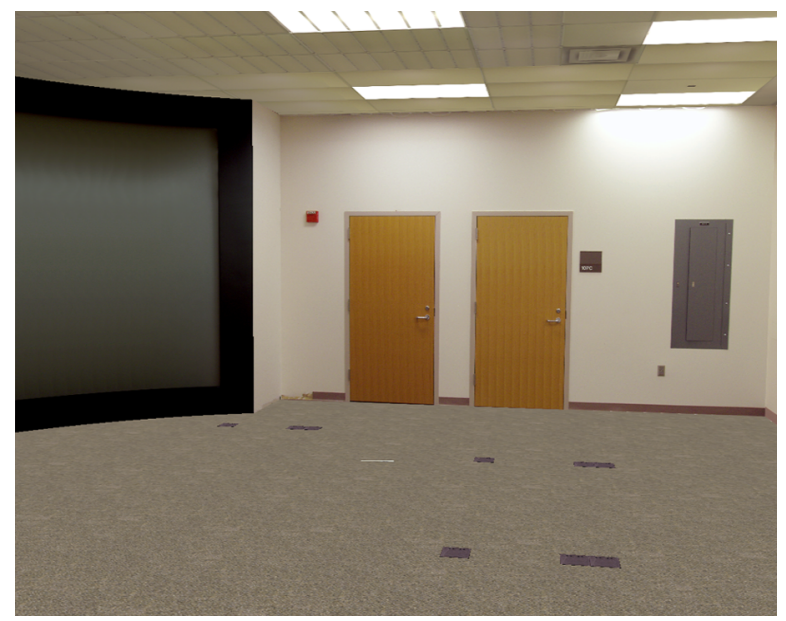

Figure 10: A screen shot of the corresponding virtual environment.

In contrast to the methodology used in our first experiment, the paths walked in the followup study were not pre-defined, but were instead determined randomly, both in their starting position and in the length of the distance interval indicated, and each path was defined and displayed independently, for each trial. In this way, we were able to ensure that participants could not carry over any information between trials or between conditions, because they had no possible opportunity to 'learn' any of the distances or paths tested.

In the virtual environment, the starting point for each distance interval was defined to be the user's current location, as indicated by the tracker, at the time of the start of each trial. The endpoint of each distance interval was defined by an automatic algorithm in which the participant's direction of gaze was used to define a vector in the floor plane, and a texture-mapped strip of virtual tape was placed at a point randomly-defined to lie between $8-25$ ' along that vector from the participant's current location. The experimenter had the option to press a key to request a new random ending tape position if $\mathrm{s} /$ he felt that the computer-defined distance interval endpoint was unsuitable (i.e. too close to an obstacle or landmark in the environment). This manual override option was used only sparingly. At a 'ready' signal from the participant, the experimenter recorded the starting point using a keypress, which also blacked out the screen. At the end of each trial, when the ending position of the participant was recorded by the experimenter, the virtual markers were deleted. As before, a 
separate keypress was used to turn the display back on when the participant had been positioned at a suitable starting point for the next trial.

In the real environment, the starting and ending points of the distance intervals were indicated by thin strips of cloth sewn to pieces of Velcro, which were interactively placed on the floor, by the experimenter, while the participant remained blindfolded. Their location was determined on an ad hoc basis, intended to mimic the random distance interval definition process used in the virtual environment condition. Both markers were surreptitiously taken up after the end of each trial, after the participant had been led (still blindfolded) away from his ending point.

\subsection{Results}

Figure 11 summarizes the results of our followup experiment in a bar chart that shows the average relative errors, (walked_distance - actual_distance) / actual_distance, computed over the 10 real world trials, and over the last 10 virtual world trials, for all participants in each condition. ${ }^{1}$ We performed a two-way mixed analysis of variance of the errors across exposure (real world first vs. virtual world first), and environment (trials done in the real world vs. trials in the virtual world), and found no significant main effects of either environment $\{\mathrm{F}(1,196)=0.06, \mathrm{p}=0.80\}$ or of exposure $\{\mathrm{F}(1,196)=0.41, \mathrm{p}=0.52\}$.

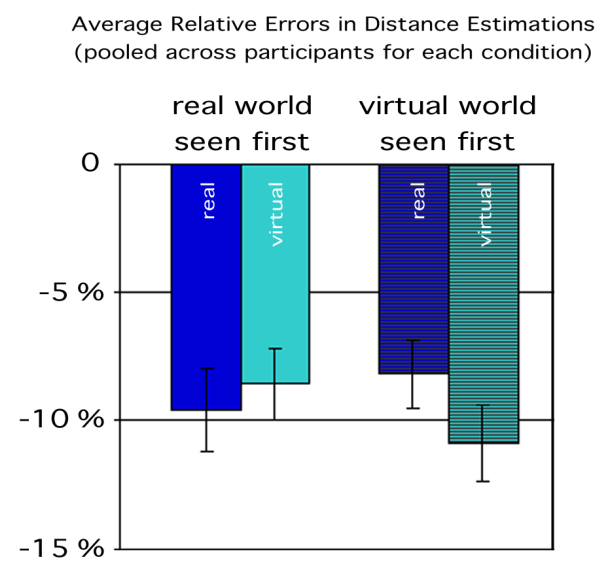

Figure 11. A bar chart showing the average relative errors in distance estimates made in the real world (dark blue) and in the matching virtual world (light blue) by participants in our followup study. The error bars represent \pm 1 standard error of the means.

Figure 12 shows separate scatterplots of all of the individual distance estimates obtained from each of our ten participants. Datapoints in each plot have been color-coded by participant number (hue) and technology (lightness), and are plotted as circles when the judgment was made in the virtual world, and as squares when the judgment was made in the real world. Trendlines, solid in the one case and dotted in the other, are superimposed to illustrate a linear fit to the data.

\subsection{Discussion}

The striking finding of our followup experiment is that, even more so than in our original experiment, we did not find indications that participants experienced a significant amount of distance compression in the immersive virtual environment, as compared

1 The choice to consider the same number of trials across all conditions was done to simplify the statistical analysis. The results using average relative errors computed over all $20 \mathrm{VE}$ trials are not significantly different, overall.

to in the real world. We did observe that our participants walked slightly 'short', on average, in both the real room and in the virtual room. However, as this phenomenon was common to both environments, we suspect that it might stem from the relative lack, in the reconfigured room as compared to the original room, of good linear perspective cues to distance, due to the room having curved rather than straight edges.

real world seen first
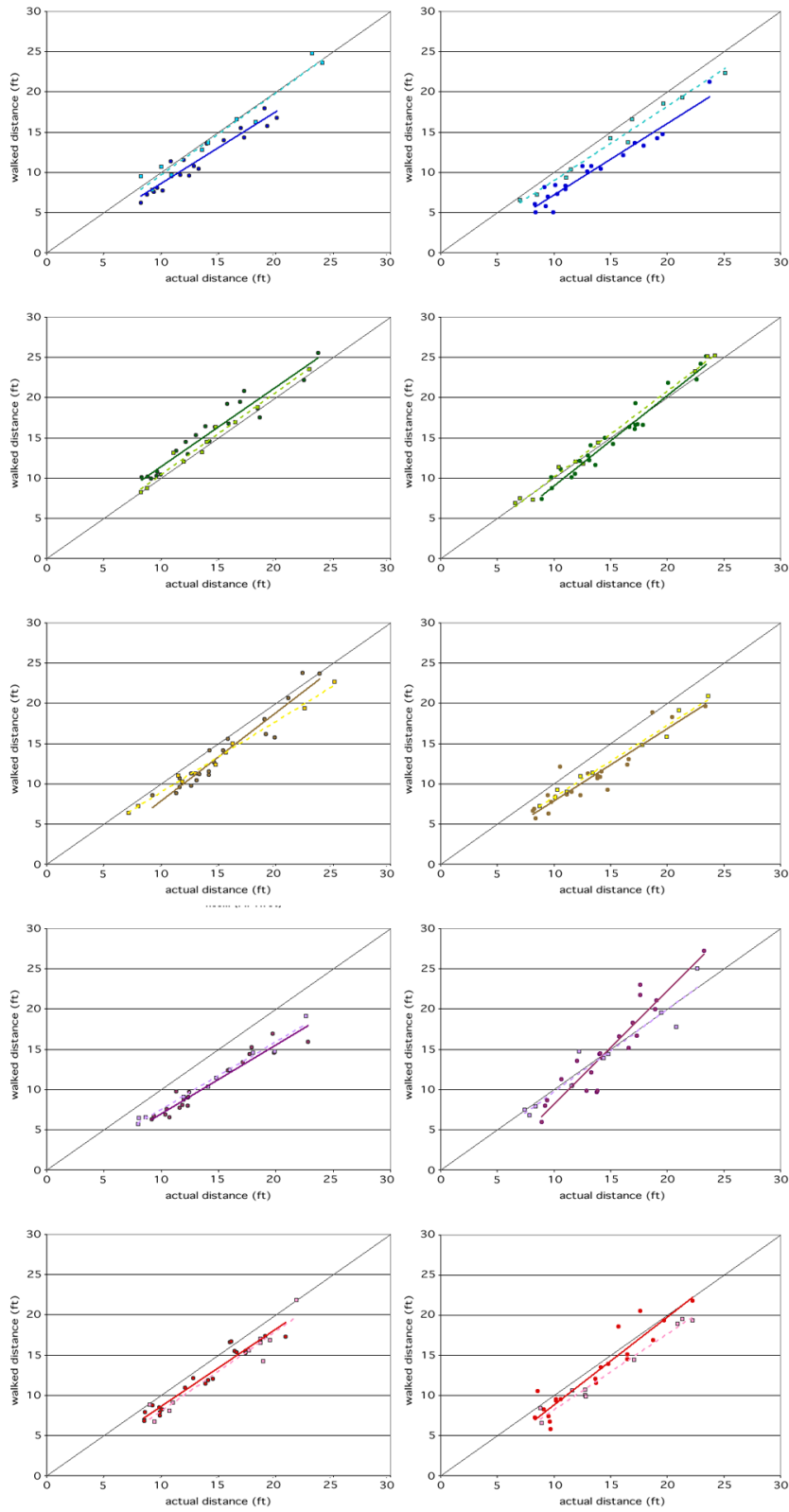

Figure 12. Individual scatter plots of distance walked, versus target distance, over all trials, for all participants.

\section{General Discussion}

Although we cannot be certain that the results in our first study were unaffected by potential methodological concerns, the fact that these results were confirmed in our second study, which was conducted under methodological conditions that cannot be faulted, leads us to believe that, in sum, we have come to a significant and interesting new discovery: namely, that in a 
situation where participants can be certain, as a result of first-hand observation, that the immersive virtual environment they are viewing identically represents the same real environment that they are physically occupying, they do not appear to exhibit the same strong indications, previously observed by others, of perceiving distances to be compressed in the virtual environment. Although, of course, the whole purpose of using virtual environments technology is to immerse participants in a different space than the one they are actually, physically, occupying, the discovery that indications of distance compression in immersive virtual environments may be significantly reduced when the spaces are known to be the same is greatly encouraging. It suggests that the problem of distance compression in immersive virtual environments may not necessarily be inherent to the technology, but may in fact stem, in significant part, from higher-level cognitive issues in the interpretation of the presented visual stimulus.

\section{FUTURE WORK}

We are intrigued by the possibility that distance perception in a virtual environment could be affected by the extent to which a person present in the VE is willing to accept that VE as being functionally equivalent [6] to the real world. If it turns out that egocentric distance compression, as indicated by an action-based metric, is, in fact, a symptom of a lack of 'presence' in an immersive virtual environment, it may become possible in the future to use such action-based metrics to quantitatively assess the extent to which a participant experiences 'immersion' in a particular virtual environment. In future work, we would like to pursue these questions more deeply, along with conducting further studies to explore strategies for facilitating an accurate perception of space and distance in immersive virtual environments that do not exactly match a person's actual occupied environment.

\section{ACKNOWLEDGMENTS}

This research was supported by the National Science Foundation through grants IIS-0313226 and CNS-0323471, by the University of Minnesota through the Digital Technology Center, and by the Linda and Ted Johnson Digital Design Consortium Endowment and Lab Set-Up Funds.

\section{REFERENCES}

[1] Lee Anderson, James Esser, and Victoria Interrante, A Virtual Environment for Conceptual Design in Architecture, In Proceedings of the 9th Eurographics Workshop on Virtual Environments/7th International Workshop on Immersive Projection Technology, 57-63, May 2003.

[2] Niels H. Bakker, Peter J. Werkhoven and Peter O. Passenier. Calibrating Visual Path Integration in VEs, Presence, 10, 2, 216-224, 2001.

[3] Sarah Creem-Regehr, Peter Willemsen, Amy A. Gooch and William B. Thompson. The influence of restricted viewing conditions on egocentric distance perception: Implications for real and virtual environments, Perception, 34, 2, 191-204, 2005.

[4] Colin G. Ellard and Sarah C. Shaughnessy. A Comparison of Visual and Nonvisual Sensory Inputs to Walked Distance in a Blind Walking Task, Perception, 32, 567-578, 2003.

[5] Digby Elliot. The Influence of Walking Speed and Prior Practice on Locomotor Distance Estimation, Journal of Motor Behavior, 19(4), 476-485, 1987.

[6] James Ferwerda. Three Varieties of Realism in Computer Graphics, Human Vision and Electronic Imaging VIII, SPIE 5007, 290-297, 2003.

[7] Sergio S. Fukusima, Jack M. Loomis and Jose A. Da Silva. Visual perception of egocentric distance as assessed by triangulation. In Journal of Experimental Psychology: Human Perception and Performance, 23, 1, 86-100, 1997.

[8] Amy A. Gooch and Peter Willemsen. Evaluating Space Perception in NPR Immersive Environments, In Proceedings of NPAR 2002 (Symposium on Non-Photorealistic Animation and Rendering), 105-110, 2002.
[9] Benjamin Lok, Samir Naik, Mary Whitton and Frederick P. Brooks, Jr Effects of Handling Real Objects and Self-Avatar Fidelity on Cognitive Task Performance and Sense of Presence in Virtual Environments, Presence, 12, 6, 615-628, 2003.

[10] Jack M. Loomis, Jose A. Da Silva, Naofumi Fujita. and Sergio S Fukusima. Visual Space Perception and Visually Directed Action, Journal of Experimental Psychology: Human Perception and Performance, 18, 4, 906-921, 1992

[11] Jack M. Loomis, Roberta L. Klatzky, Reginald G. Golledge, Joseph G. Cicinelli, James W. Pellegrino and Phyllis A. Fry. Nonvisual Navigation by Blind and Sighted: Assessment of Path Integration Ability, Journal of Experimental Psychology: General, 122, 1, 73-91, 1993.

[12] Jack M. Loomis and Joshua M. Knapp. Visual Perception of Egocentric Distance in Real and Virtual Environments. In Virtual and Adaptive Environments, L.J. Hettinger and M.W. Haas, Eds. Mahwah, NJ, 21-45, 2003

[13] Joshua M. Knapp and Jack M. Loomis. Limited Field of View of HeadMounted Displays Is Not the Cause of Distance Underestimation in Virtual Environments. In Presence: : Teleoperators and Virtual Environments, 13, 5, 572-577, 2004.

[14] Ross Messing and Frank H. Durgin. Distance Perception and the Visual Horizon in Head-Mounted Displays, ACM Transactions on Applied Perception, 2, 3, 234-250, 2005.

[15] Christopher C. Pagano and Geoffrey P. Bingham. Comparing measures of monocular distance perception: verbal and reaching errors are not correlated, Journal of Experimental Psychology: Human Perception and Performance, 24, 4, 1037-1051, 1998.

[16] Jodie M. Plumert, Joseph K. Kearney and James F. Cremer. Distance Perception in Real and Virtual Environments. In Proceedings of the First Symposium on Applied Perception in Graphics and Visualization, 27-34, August 2004.

[17] John J. Rieser, Daniel H. Ashmead, Charles R. Taylor and Grant A. Youngquist. Visual Perception and the Guidance of Locomotion Without Vision to Previously Seen Targets, Perception, 19, 675-689, 1990.

[18] Cynthia S. Sahm, Sarah Creem-Regehr, William B. Thompson and Peter Willemsen. Throwing vs. Walking as Indicators of Distance Perception in Real and Virtual Environments, ACM Transactions on Applied Perception, 2, 1, 35-45, 2005 .

[19] Michael J. Sinai, William K. Krebs, Rudy P. Darken, J.H. Rowland and J.S. McCarley. Egocentric Distance Perception in a Virtual Environment Using a Perceptual Matching Task, Human Factors and Ergonomics Society $43^{\text {rd }}$ Annual Meeting, 43, 1256-1260, 1999.

[20]Runa E. Steenhuis and Melvyn A. Goodale. The Effects of Time and Distance on Accuracy of Target-Directed Locomotion: Does an Accurate Short-Term Memory for Spatial Location Exist?, Journal of Motor Behavior, 20, 4, 399-415, 1988.

[21] William B. Thompson, Peter Willemsen, Amy A. Gooch, Sarah H. CreemRegehr, Jack M. Loomis and Andrew C. Beall. Does the Quality of the Computer Graphics Matter When Judging Distances in Visually Immersive Environments? Presence: Teleoperators and Virtual Environments, 13, 5, 560-571, 2004.

[22] James A. Thomson. Is Continuous Visual Monitoring Necessary in Visually Guided Locomotion?, Journal of Experimental Psychology: Human Perception and Performance, 9, 427-443, 1983.

[23] Benjamin A. Watson and Larry F. Hodges. Using Texture Maps to Correct for Optical Distortion in Head-Mounted Displays. In Proceedings of the IEEE Virtual Reality Annual International Symposium, 172-178, 1995,

[24] Peter Willemsen and Amy A. Gooch. Perceived Egocentric Distances in Real, Image-Based, and Traditional Virtual Environments, In Proceedings of the IEEE Virtual Reality 2002 (VR'02), 275-276.

[25] Peter Willemsen, Mark B. Colton, Sarah Creem-Regehr,and William B. Thompson. The Effects of Head-Mounted Display Mechanics on Distance Judgments in Virtual Environments. In Proceedings of the First ACM/SIGGRAPH Symposium on Applied Perception in Graphics and Visualization, 35-38, 2004.

[26] Bob G. Witmer and Paul B. Kline. Judging Perceived and Traversed Distance in Virtual Environments, Presence: Teleoperators and Virtual Environments, 7, 2, 144-167, 1998.

[27] Bob G. Witmer and Wallace J. Sadowski. Nonvisually Guided Locomotion to a Previously Viewed Target in Real and Virtual Environments, Human Factors, 40, i3, 478-484, 1998. 